\title{
Viscosity iterative method for split common null point prob- lems and fixed point problems
}

\author{
Yanlai Song*, Xinhong Chen \\ College of Science, Zhongyuan University of Technology, 450007 Zhengzhou, China.
}

\begin{abstract}
In this paper, we introduce and study an Ishikawa-like iterative algorithm to approximate a common solution of a split common null point problem and a fixed point problem of asymptotically pseudo-contractive mappings in the intermediate sense on unbounded domains. We prove that the sequence generated by the iterative scheme strongly converges to a common solution of the above-said problems. The method in this paper is novel and different from those given in many other papers. The results are the extension and improvement of the recent results in the literature.
\end{abstract}

Keywords: Banach space, split common null point problem, fixed point, metric resolvent, asymptotically pseudocontractive mapping in the intermediate sense.

2010 MSC: 47H09, 47H10, 49H17.

(C)2019 All rights reserved.

\section{Introduction}

Let $\mathrm{H}$ be a real Hilbert space, $\mathrm{C}$ a nonempty closed convex subset of $\mathrm{H}, \mathrm{T}: \mathrm{C} \rightarrow \mathrm{C}$ a self-mapping of $\mathrm{C}$ and $\operatorname{Fix}(T):=\{x \in C: T x=x\}$.

Recall that a mapping $T$ is said to be an asymptotically $k$-strict pseudo-contraction [10], if there exist a constant $k \in[0,1)$ and a sequence $\left\{k_{n}\right\} \subset[1,+\infty)$ with $k_{n} \rightarrow 1$ as $n \rightarrow \infty$ such that

$$
\left\|T^{n} x-T^{n} y\right\|^{2} \leqslant k_{n}\|x-y\|^{2}+k\left\|\left(I-T^{n}\right) x-\left(I-T^{n}\right) y\right\|^{2}, \quad \forall n \geqslant 1, x, y \in C .
$$

Recall that $\mathrm{T}$ is said to be an asymptotically k-strict pseudo-contraction in the intermediate sense [18], if

$$
\limsup _{n \rightarrow \infty} \sup _{x, y \in C}\left\{\left\|T^{n} x-T^{n} y\right\|^{2}-k_{n}\|x-y\|^{2}-k\left\|\left(I-T^{n}\right) x-\left(I-T^{n}\right) y\right\|^{2}\right\} \leqslant 0,
$$

where $k \in[0,1)$ and $k_{n} \in[1, \infty)$ such that $k_{n} \rightarrow 1$ as $n \rightarrow \infty$. Put

$$
\tau_{n}=\max \left\{\sup _{x, y \in C}\left(\left\|T^{n} x-T^{n} y\right\|^{2}-k_{n}\|x-y\|^{2}-k\left\|\left(I-T^{n}\right) x-\left(I-T^{n}\right) y\right\|^{2}\right), 0\right\} .
$$

\footnotetext{
${ }^{*}$ Corresponding author
}

Email addresses: songyanlai2009@163.com (Yanlai Song), chenxh6@163.com (Xinhong Chen)

doi: $10.22436 /$ jnsa.012.02.03

Received: 2017-07-25 Revised: 2018-07-25 Accepted: 2018-09-21 
It follows that $\tau_{n} \rightarrow 0$ as $n \rightarrow \infty$. Then, (1.1) is reduced to the following:

$$
\left\|T^{n} x-T^{n} y\right\|^{2} \leqslant k_{n}\|x-y\|^{2}+k\left\|\left(I-T^{n}\right) x-\left(I-T^{n}\right) y\right\|^{2}+\tau_{n}, \quad \forall n \geqslant 1, x, y \in C .
$$

A mapping $T$ is said to be an asymptotically pseudo-contraction [19], if there exists a sequence $\left\{k_{n}\right\} \subset$ $[1,+\infty)$ with $k_{n} \rightarrow 1$ as $n \rightarrow \infty$ such that

$$
\left\langle T^{n} x-T^{n} y, x-y\right\rangle \leqslant k_{n}\|x-y\|^{2}, \quad \forall n \geqslant 1, x, y \in C .
$$

Recall that $\mathrm{T}$ is said to be an asymptotically pseudo-contraction in the intermediate sense [16], if

$$
\limsup _{n \rightarrow \infty} \sup _{x, y \in C}\left\{\left\langle T^{n} x-T^{n} y, x-y\right\rangle-k_{n}\|x-y\|^{2}\right\} \leqslant 0,
$$

where $k_{n} \in[1, \infty)$ such that $k_{n} \rightarrow 1$ as $n \rightarrow \infty$. Put

$$
v_{n}=\max \left\{\sup _{x, y \in C}\left(\left\langle T^{n} x-T^{n} y, x-y\right\rangle-k_{n}\|x-y\|^{2}\right), 0\right\} .
$$

It follows that $v_{n} \rightarrow 0$ as $n \rightarrow \infty$. Then, (1.3) is reduced to the following:

$$
\left\langle T^{n} x-T^{n} y, x-y\right\rangle \leqslant k_{n}\|x-y\|^{2}+v_{n}, \quad \forall n \geqslant 1, x, y \in C .
$$

In real Hilbert spaces, we see that (1.4) is equivalent to

$$
\left\|T^{n} x-T^{n} y\right\|^{2} \leqslant\left(2 k_{n}-1\right)\|x-y\|^{2}+\left\|\left(I-T^{n}\right) x-\left(I-T^{n}\right) y\right\|^{2}+2 v_{n}, \quad \forall n \geqslant 1, x, y \in C .
$$

Remark 1.1. From above definitions, we find that

- an asymptotically k-strict pseudocontraction is a uniformly L-Lipschitz mapping with $L=\frac{k+\sqrt{1+\left(\sup \left\{k_{n}\right\}-1\right)(1-k)}}{1-k}$; see [9].

- if $T$ is an asymptotically k-strict pseudocontraction in the intermediate sense, then it is an asymptotically pseudocontractive mapping in the intermediate sense but the converse does not hold such as in the following example.

Example 1.2 ([7]). Let $H=\mathbb{R}$ and $C=[0,1]$. For all $x \in C$, define $T: C \rightarrow C$ by

$$
\mathrm{T} x=(1-\sqrt{x})^{2} .
$$

Then $T$ is an asymptotically pseudocontractive mapping in the intermediate sense but not an asymptotically k-strict pseudocontraction in the intermediate sense.

Recall that $\mathrm{T}$ is said to be uniformly asymptotically regular [27], if for any $\varepsilon>0$, there exists an integer $N \geqslant 1$ such that

$$
\left\|T^{n+1} x-T^{n} x\right\|^{2} \leqslant \varepsilon, \quad \forall n \geqslant N, x \in C .
$$

Clearly, (1.6) is equivalent to the following

$$
\lim _{n \rightarrow \infty} \sup _{x \in C}\left\{\left\|T^{n+1} x-T^{n} x\right\|\right\}=0 .
$$

A mapping $T$ is said to be uniformly L-Lipschitz [27], if there exists some $L>0$ such that

$$
\left\|T^{n} x-T^{n} y\right\| \leqslant L\|x-y\|, \quad \forall n \geqslant 1, x, y \in C .
$$

Recall that $\mathrm{T}$ is said to be firmly nonexpansive if

$$
\|T x-T y\|^{2} \leqslant\langle T x-T y, x-y\rangle, \quad \forall x, y \in C .
$$


It is easily found that $\mathrm{T}$ is firmly nonexpansive if and only if $\mathrm{T}=\frac{1}{2}(\mathrm{I}+\mathrm{V})$ for some nonexpansive mapping $\mathrm{V}$; hence a firmly nonexpansive mapping must be nonexpansive.

The class of asymptotically pseudo-contractive mappings in the intermediate sense was introduced by Qin et al. [16]. We know that the class of asymptotically pseudo-contractive mappings in the intermediate sense contains properly the class of asymptotic pseudo-contractions as a subclass. To compute the fixed point of asymptotically pseudo-contractive mappings in the intermediate sense, Qin et al. [16] demonstrated the hybrid methods on bounded domains. But his methods cannot be applied to the case when domains are unbounded.

Motivated by Qin et al. [16], Ge [7] introduced and studied certain new hybrid algorithm with variable coefficients for asymptotically pseudo-contractive mappings in the intermediate sense on unbounded domains in real Hilbert spaces. However, we find that the sequence generated by the algorithm in [7] is difficult to compute because it involves projecting $x_{0}$ onto the intersection of closed convex sets $C_{n}$ and $Q_{n}$ for each $n \geqslant 1$.

Recently, Zegeye et al. [26] studied the convergence for the class of asymptotically pseudocontractive mappings in the intermediate sense without using the hybrid method adopted by Qin et al. [16] and Ge [7]. They presented the following algorithm

$$
\left\{\begin{array}{l}
y_{n}=\left(1-\beta_{n}\right) x_{n}+\beta_{n} T^{n} x_{n}, \\
x_{n+1}=\left(1-\alpha_{n}\right) x_{n}+\alpha_{n} T^{n} y_{n}, \quad n \geqslant 1,
\end{array}\right.
$$

where $\mathrm{T}: \mathrm{C} \rightarrow \mathrm{C}$ is uniformly L-Lipschitz and asymptotically pseudo-contractive in the intermediate sense with sequences $\left\{k_{n}\right\} \subset[1, \infty)$ and $\left\{v_{n}\right\} \subset[0, \infty)$ as defined in (1.4). They also proved a strong convergence theorem under assumption that the interior of $\operatorname{Fix}(\mathrm{T})$ is nonempty. But we observe that the assumption that the interior of $\operatorname{Fix}(T)$ is nonempty is also a severe restriction. It is trivial to see the fact from Example 1.3.

Example 1.3 ([7]). Let $H=\mathbb{R}$ and $C=[1,9]$. For all $x \in C$, define $T: C \rightarrow C$ by

$$
\mathrm{T} x=(4-\sqrt{x})^{2} .
$$

Then $T$ is a uniformly L-Lipschitz mapping and asymptotically pseudo-contractive mapping in the intermediate sense. It is easy to see that $\operatorname{Fix}(T)=4$ and the interior of $\operatorname{Fix}(T)$ is empty.

On the other hand, Takahashi and Yao [22] considered the following split common null point problem in Banach spaces: Given set-valued mappings $\mathrm{A}: \mathrm{H} \rightarrow 2^{\mathrm{H}}, \mathrm{B}: \mathrm{E} \rightarrow 2^{\mathrm{E}^{*}}$, respectively, and bounded linear operator $\mathrm{T}: \mathrm{H} \rightarrow \mathrm{E}$, the split common null point problem [22] is to find a point $z \in \mathrm{H}$ such that

$$
z \in A^{-1} 0 \bigcap \mathrm{T}^{-1}\left(\mathrm{~B}^{-1} 0\right) \neq \emptyset
$$

where $A^{-1} 0$ and $B^{-1} 0$ are null point sets of $A$ and $B$, respectively. In order to find a solution of the split common null point problem in Banach spaces, they also introduced the following hybrid method in mathematical programming:

$$
\left\{\begin{array}{l}
z_{n}=J_{\lambda_{n}}\left(x_{n}-\lambda_{n} T^{*} J_{E}\left(T x_{n}-Q_{\mu_{n}} T x_{n}\right)\right), \\
y_{n}=\alpha_{n} z_{n}+\left(1-\alpha_{n}\right) x_{n} \\
C_{n}=\left\{z \in H:\left\|y_{n}-u\right\| \leqslant\left\|x_{n}-u\right\|,\right. \\
Q_{n}=\left\{z \in H:\left\langle x_{n}-z, x_{1}-x_{n}\right\rangle \geqslant 0\right\} \\
x_{n+1}=P_{C_{n} \cap Q_{n}} x_{1}, \quad \forall n \in \mathbb{N} .
\end{array}\right.
$$

Then they obtained a strong convergence theorem under some mild restrictions on the parameters.

Motivated and inspired by Takahashi and Yao [22], Qin et al. [16], Ge [7], Zegeye et al. [26] and Olaleru and Okeke [14], we suggest and analyze a new iterative algorithm for finding a common solution to the split common null point problem and the fixed point problem of asymptotically pseudo-contractive 
mappings in the intermediate sense. We prove the convergence of the proposed algorithm without using hybrid method. Also the assumption that the interior of $\operatorname{Fix}(T)$ is empty is not required. Our results improve essentially the corresponding results in $[7,14,16,22,26]$. Further, some other results are also improved; see $[5,6,8,13,15,17-19,24,25]$.

\section{Preliminaries}

Throughout this paper, we denote $E$ the real Banach space, $E^{*}$ the dual of $E$, I the identity mapping on $E, H$ the real Hilbert space, $\mathbb{R}$ the set of real numbers, and $\mathbb{N}$ the set of nonnegative integers. The expressions $x_{n} \rightarrow x$ and $x_{n} \rightarrow x$ denote the strong and weak convergence of the sequence $\left\{x_{n}\right\}$, respectively. The (normalized) duality mapping of $E$ is denoted by J, that is,

$$
J x=\left\{x^{*} \in E^{*}:\left\langle x, x^{*}\right\rangle=\|x\|^{2},\left\|x^{*}\right\|=\|x\|\right\}
$$

for all $x \in E$, where $\langle\cdot, \cdot\rangle$ denotes the generalized duality pairing between $E$ and $E^{*}$. If $E$ is a Hilbert space, then $\mathrm{J}=\mathrm{I}$, where $\mathrm{I}$ is the identity mapping on $\mathrm{H}$.

The norm of a Banach space $E$ is said to be Gâteaux differentiable if the limit

$$
\lim _{t \rightarrow 0} \frac{\|x+t y\|-\|x\|}{t}
$$

exists for all $x, y$ on the unit sphere $S(E)=\{x \in E:\|x\|=1\}$. In this case, we say that $E$ is smooth. In a smooth Banach space $E$, it holds that

$$
(\|x\|-\|y\|)^{2} \leqslant\langle x-y, J x-J y\rangle
$$

for all $x, y \in E$; see [1].

The norm of $E$ is said to be uniformly Gâteaux differentiable if for each $y \in S(E)$, the limit (2.1) is attained uniformly for $x \in S(E)$. The norm of $E$ is said to be Fréchet differentiable if for each $x \in S(E)$, the limit (2.1) is attained uniformly for $y \in S(E)$. The norm of $E$ is said to be uniformly Fréchet differentiable if the limit (2.1) is attained uniformly for $x, y \in S(E)$. In this case $E$ is said to be uniformly smooth. It is known that:

- if $\mathrm{E}$ is smooth, then the duality mapping J is single-valued;

- if the norm of $E$ is uniformly Gâteaux differentiable, then J is uniformly norm-to-weak* continuous on each bounded subset of $E$;

- if the norm of $E$ is Fréchet differentiable, then $J$ is norm-to-norm continuous;

- if $E$ is uniformly smooth, then $J$ is uniformly norm-to-norm continuous on each bounded subset of E;

see $[20,21]$ for more details.

A Banach space $E$ is said to be strictly convex if $\|x-y\|<2$ whenever $x, y \in S(E)$ and $x \neq y$. It is known that if $E$ is strictly convex, then the duality mapping $J$ is injective, that is, $x, y \in E$ and $x \neq y$ imply $J x \cap J y=\emptyset$. A Banach space $E$ is said to be uniformly convex if $\left\|x_{n}-y_{n}\right\| \rightarrow 0$ whenever $\left\{x_{n}\right\}$ and $\left\{y_{n}\right\}$ are sequences in $S(E)$ and $\left\|x_{n}+y_{n}\right\| \rightarrow 2$. It is known that if $E$ is uniformly convex, then

- $E$ is strictly convex and reflexive;

- E has the Kadec-Klee property, that is, a sequence $\left\{x_{n}\right\}$ in E converges strongly to $x$ whenever $x_{n} \rightarrow x$ and $\left\|x_{n}\right\| \rightarrow\|x\| ;$

see $[20,21]$ for more details.

We know that $E$ is reflexive if and only if $J$ is surjective. Therefore, if $E$ is a smooth, strictly convex and reflexive Banach space, then $J$ is a single-valued bijection and in this case, the inverse mapping $\mathrm{J}^{-1}$ coincides with the duality mapping $\mathrm{J}^{*}$ on $\mathrm{E}^{*}$. 
Let $E$ be a Banach space and let $A$ be a mapping of $E$ into $2^{E^{*}}$. The effective domain of $A$ is denoted by $\operatorname{dom}(A)$, that is, $\operatorname{dom}(A)=\{x \in E: A x \neq \emptyset\}$. A multi-valued mapping $A$ on $E$ is said to be monotone if $\left\langle x-y, u^{*}-v^{*}\right\rangle \geqslant 0$ for all $x, y \in \operatorname{dom}(A), u^{*} \in A x$, and $v^{*} \in A y$. A monotone operator $A$ on $E$ is said to be maximal if its graph is not properly contained in the graph of any other monotone operator on $E$. Suppose $E$ is a smooth, strictly convex, and reflexive Banach space and $A$ is a monotone operator. Then it is known that the single-valued mapping $J_{r}:=\left(I+r J^{-1} A\right)^{-1}$ is well-defined for all $r>0$; see [4]. Such $\mathrm{J}_{\mathrm{r}}, \mathrm{r}>0$ are called the metric resolvents of $A$. It is also known that if $A$ is a maximal monotone operator, then

$$
\operatorname{ran}\left(\mathrm{I}+\mathrm{rJ} \mathrm{J}^{-1} \mathrm{~A}\right)=\mathrm{E}
$$

for all $r>0$, where $\operatorname{ran}\left(I+r J^{-1} A\right)$ is the range of $\left(I+r J^{-1} A\right)$; see [20]. The set of null points of $A$ is defined by $A^{-1} 0=\{z \in E: 0 \in A z\}$. We know that $A^{-1} 0$ is closed and convex and $\operatorname{Fix}\left(J_{r}\right)=A^{-1} 0$; see $[2,20]$.

Let $\mathrm{H}$ be a Hilbert space and let $A$ be a maximal monotone operator on a Hilbert space $H$. The metric resolvent $J_{r}$ of $A$ is called the resolvent of $A$ simply. It is known that the resolvent $J_{r}$ of $A$ for any $r>0$ is firmly nonexpansive, i.e.,

$$
\left\|J_{r} x-J_{r} y\right\|^{2} \leqslant\left\langle x-y, J_{r} x-J_{r} y\right\rangle, \quad \forall x, y \in H .
$$

Lemma 2.1. In a Hilbert space $\mathrm{H}$, it holds that

$$
\|\lambda x+(1-\lambda) y\|^{2}=\lambda\|x\|^{2}+(1-\lambda)\|y\|^{2}-\lambda(1-\lambda)\|x-y\|^{2}
$$

for all $x, y \in \mathrm{H}$ and $\lambda \in[0,1]$, and

$$
2\langle x-y, u-v\rangle \leqslant\|x-v\|^{2}+\|y-u\|^{2}-\|x-u\|^{2}-\|y-v\|^{2}
$$

for all $x, y, u, v \in \mathrm{H}$.

Lemma 2.2. There holds the following inequality in an inner product space $\mathrm{H}$ :

$$
\|x+y\|^{2} \leqslant\|x\|^{2}+2\langle y, x+y\rangle, \quad \forall x, y \in H .
$$

Lemma 2.3 ([12]). Let $\left\{\alpha_{n}\right\}$ be a sequence of real numbers such that there exists a subsequence $\left\{n_{i}\right\}$ of $\{n\}$ such that $\alpha_{n_{i}}<\alpha_{n_{i}+1}$ for all $i \in \mathbb{N}$. Then there exists a nondecreasing sequence $\left\{m_{k}\right\} \subseteq \mathbb{N}$ such that $m_{k} \rightarrow \infty$ and the following properties are satisfied for all (sufficiently large) numbers $\mathrm{k} \in \mathbb{N}$ :

$$
\alpha_{m_{k}} \leqslant \alpha_{m_{k}+1} \text { and } \alpha_{k} \leqslant \alpha_{m_{k}+1} .
$$

In fact, $m_{k}=\max \left\{j \leqslant k: \alpha_{j}<\alpha_{j+1}\right\}$.

Lemma 2.4 ([23]). Let $\left\{\alpha_{\mathrm{n}}\right\}$ be a sequence of nonnegative real numbers satisfying the following relation:

$$
\alpha_{n+1} \leqslant\left(1+\gamma_{n}\right) \alpha_{n}+\sigma_{n}, \quad n \geqslant n_{0},
$$

where $\mathrm{n}_{0}$ is some nonnegative integer. If $\sum_{n=1}^{\infty} \gamma_{n}<\infty$ and $\sum_{n=1}^{\infty} \sigma_{n}<\infty$, then $\lim _{n \rightarrow \infty} \alpha_{n}$ exists.

Lemma 2.5 ([16]). Let $\mathrm{C}$ be a nonempty close convex subset of a real Hilbert space $\mathrm{H}$ and $\mathrm{T}: \mathrm{C} \rightarrow \mathrm{C}$ a uniformly L-Lipschitz and asymptotically pseudo-contractive mapping in the intermediate sense such that $\operatorname{Fix}(\mathrm{T})$ is nonempty. Then $\mathrm{I}-\mathrm{T}$ is demiclosed at zero.

Lemma 2.6 ([16]). Let $\mathrm{C}$ be a nonempty close convex subset of a real Hilbert space $\mathrm{H}$ and $\mathrm{T}: \mathrm{C} \rightarrow \mathrm{C}$ a uniformly L-Lipschitz and asymptotically pseudo-contractive mapping in the intermediate sense with sequences $\left\{k_{n}\right\}$ and $\left\{v_{n}\right\}$ as defined in (1.4). Then Fix (T) is a closed convex subset of $\mathrm{C}$. 
Lemma 2.7 ([11]). Let $\left\{\alpha_{n}\right\}$ be a sequence of nonnegative numbers satisfying the property:

$$
\alpha_{n+1} \leqslant\left(1-\gamma_{n}\right) \alpha_{n}+b_{n}+\gamma_{n} c_{n}, \quad n \in \mathbb{N},
$$

where $\left\{\gamma_{n}\right\},\left\{b_{n}\right\},\left\{c_{n}\right\}$ satisfy the restrictions:

(i) $\sum_{n=1}^{\infty} \gamma_{n}=\infty, \quad \lim _{n \rightarrow \infty} \gamma_{n}=0$;

(ii) $b_{n} \geqslant 0, \quad \sum_{n=1}^{\infty} b_{n}<\infty$;

(iii) $\limsup _{n \rightarrow \infty} c_{n} \leqslant 0$.

Then, $\lim _{n \rightarrow \infty} \alpha_{n}=0$.

\section{Main results}

We also need the following lemmas which are fundamental for Theorem 3.4.

Lemma 3.1. Let $\mathrm{H}$ be a Hilbert space and let $\mathrm{E}$ be a uniformly convex and smooth Banach space. Let $\mathrm{J}$ be the duality mapping on $\mathrm{E}$. Let $\mathrm{A}$ and $\mathrm{B}$ be maximal monotone operators of $\mathrm{H}$ into $2^{\mathrm{H}}$ and $\mathrm{E}$ into $2^{\mathrm{E}}$, respectively. Let $\mathrm{J}_{\lambda}$ be the resolvent of $\mathrm{A}$ for $\lambda>0$ and let $\mathrm{Q}_{\mu}$ be the metric resolvent of $\mathrm{B}$ for $\mu>0$. Let $\mathrm{S}: \mathrm{H} \rightarrow \mathrm{E}$ be a bounded linear operator such that $\mathrm{S} \neq 0$ and let $\mathrm{S}^{*}$ be the adjoint operator of $\mathrm{S}$. Suppose that $\mathrm{A}^{-1} 0 \cap \mathrm{S}^{-1}\left(\mathrm{~B}^{-1} 0\right) \neq \emptyset$. Then it holds, for all $\lambda, r>0$, that

$$
\operatorname{Fix}\left(J_{\lambda}\left(I-r S^{*} J\left(I-Q_{\mu}\right) S\right)\right)=A^{-1} 0 \bigcap S^{-1}\left(B^{-1} 0\right) .
$$

Proof. We first prove that

$$
\operatorname{Fix}\left(J_{\lambda}\left(I-r S^{*} J\left(I-Q_{\mu}\right) S\right)\right) \subseteq A^{-1} 0 \bigcap S^{-1}\left(B^{-1} 0\right) .
$$

Suppose that $z=\mathrm{J}_{\lambda}\left(\mathrm{I}-\mathrm{r} \mathrm{S}^{*} \mathrm{~J}\left(\mathrm{I}-\mathrm{Q}_{\mu}\right) \mathrm{S}\right) z$ and $z_{0} \in \mathrm{A}^{-1} 0 \cap \mathrm{S}^{-1}\left(\mathrm{~B}^{-1} 0\right)$. Then we have

$$
z=J_{\lambda}\left(I-r S^{*} J\left(I-Q_{\mu}\right) S\right) z \Leftrightarrow\left(I-r S^{*} J\left(I-Q_{\mu}\right) S\right) z \in(I+\lambda A) z \Leftrightarrow-\frac{r}{\lambda} S^{*} J\left(I-Q_{\mu}\right) S z \in A z .
$$

Since $A$ is monotone and $0 \in A z_{0}$, we see that

$$
\left\langle z-z_{0}, S^{*} J\left(I-Q_{\mu}\right) S z\right\rangle \leqslant 0
$$

and hence

$$
\left\langle\mathrm{S} z-\mathrm{S} z_{0}, \mathrm{~J}\left(\mathrm{I}-\mathrm{Q}_{\mu}\right) \mathrm{S} z\right\rangle \leqslant 0 .
$$

On the other hand, since B is monotone and $S z_{0} \in \mathrm{B}^{-1}(0)$, we have from [2] (or [22]) that

$$
\left\langle\mathrm{Q}_{\mu} \mathrm{S} z-\mathrm{S} z_{0}, \mathrm{~J}\left(\mathrm{I}-\mathrm{Q}_{\mu}\right) \mathrm{S} z\right\rangle \geqslant 0 .
$$

Adding up (3.1) and (3.2) we find that

$$
\left\|S z-Q_{\mu} S z\right\|^{2}=\left\langle S z-Q_{\mu} S z, J\left(I-Q_{\mu}\right) S z\right\rangle \leqslant 0 .
$$

Therefore, $S z=Q_{\mu} S z$. That is, $z \in S^{-1}\left(B^{-1} 0\right)$ and also $S z-Q_{\mu} S z=0$ implying $\left.S^{*} J\left(I-Q_{\mu}\right) S\right) z=0$. This reduces the fixed point equation $z=\mathrm{J}_{\lambda}\left(\mathrm{I}-\mathrm{rS} \mathrm{S}^{*} \mathrm{~J}\left(\mathrm{I}-\mathrm{Q}_{\mu}\right) \mathrm{S}\right) z$ to the fixed point equation $z=\mathrm{J}_{\lambda} z$ that is equivalent to $z \in A^{-1} 0$. Consequently, $z \in A^{-1} 0 \bigcap S^{-1}\left(B^{-1} 0\right)$.

We now prove

$$
A^{-1} 0 \bigcap S^{-1}\left(B^{-1} 0\right) \subseteq \operatorname{Fix}\left(J_{\lambda}\left(I-r S^{*} J\left(I-Q_{\mu}\right) S\right)\right) .
$$

Since $z_{0} \in A^{-1} 0 \bigcap S^{-1}\left(B^{-1} 0\right)$, we have that $z_{0} \in A^{-1} 0$ and $z_{0} \in S^{-1}\left(B^{-1} 0\right)$. It follows that $z_{0}=\mathrm{J}_{\lambda} z_{0}$ and $\mathrm{S} z_{0}=\mathrm{Q}_{\mu} \mathrm{S} z_{0}$. Hence, we have

$$
z_{0}=\mathrm{J}_{\lambda}\left(\mathrm{I}-\mathrm{rS} \mathrm{S}^{*} \mathrm{~J}\left(\mathrm{I}-\mathrm{Q}_{\mu}\right) \mathrm{S}\right) z_{0},
$$

which implies $A^{-1} 0 \bigcap S^{-1}\left(B^{-1} 0\right) \subseteq \operatorname{Fix}\left(J_{\lambda}\left(I-r S^{*} J\left(I-Q_{\mu}\right) S\right)\right)$. This completes the proof. 
Lemma 3.2. In the setting of Lemma 3.1, if $\mathrm{r} \in\left(0, \frac{2}{\|\mathrm{~S}\|^{2}}\right]$, then the mapping $\mathrm{J}_{\lambda}\left(\mathrm{I}-\mathrm{rS} \mathrm{S}^{*}\left(\mathrm{I}-\mathrm{Q}_{\mu}\right) \mathrm{S}\right): \mathrm{H} \rightarrow \mathrm{H}$ is quasi-nonexpansive.

Proof. Since the resolvent $J_{\lambda}$ is nonexpansive, we see that, for $u \in A^{-1} 0 \bigcap S^{-1}\left(B^{-1} 0\right)$,

$$
\begin{aligned}
& \left\|J_{\lambda}\left(z-r S^{*} J\left(S z-Q_{\mu} S z\right)\right)-u\right\|^{2} \\
& \leqslant\left\|z-r S^{*} J\left(S z-Q_{\mu} S z\right)-u\right\|^{2} \\
& =\|z-u\|^{2}+\left\|r S^{*} J\left(S z-Q_{\mu} S z\right)\right\|^{2}-2 r\left\langle z-u, S^{*} J\left(S z-Q_{\mu} S z\right)\right\rangle \\
& \leqslant\|z-u\|^{2}+r^{2}\|S\|^{2}\left\|S z-Q_{\mu} S z\right\|^{2}-2 r\left\langle S z-Q_{\mu} S z+Q_{\mu} S z-S u, J\left(S z-Q_{\mu} S z\right)\right\rangle \\
& \leqslant\|z-u\|^{2}+r^{2}\|S\|^{2}\left\|S z-Q_{\mu} S z\right\|^{2}-2 r\left\|S z-Q_{\mu} S z\right\|^{2}-2 r\left\langle Q_{\mu} S z-S u, J\left(S z-Q_{\mu} S z\right)\right\rangle \\
& \leqslant\|z-u\|^{2}+r^{2}\|S\|^{2}\left\|S z-Q_{\mu} S z\right\|^{2}-2 r\left\|S z-Q_{\mu} S z\right\|^{2} \\
& =\|z-u\|^{2}-r\left(2-r\|S\|^{2}\right)\left\|S z-Q_{\mu} S z\right\|^{2} \\
& \leqslant\|z-u\|^{2} .
\end{aligned}
$$

This shows that the mapping $\mathrm{J}_{\lambda}\left(z-r \mathrm{~S}^{*} \mathrm{~J}\left(\mathrm{~S} z-\mathrm{Q}_{\mu} \mathrm{Sz}\right)\right)$ is quasi-nonexpansive.

Lemma 3.3 ([3]). Let $\mathrm{H}$ be a Hilbert space and let $\mathrm{A}$ be a maximal monotone operator with resolvent $\mathrm{J}_{\lambda}=$ $(I+\lambda A)^{-1}$ for $\lambda>0$. Then it holds, for all $\lambda, \mu>0$ and $x \in H$,

$$
\mathrm{J}_{\lambda} x=\mathrm{J}_{\mu}\left(\frac{\mu}{\lambda} x+\left(1-\frac{\mu}{\lambda}\right) \mathrm{J}_{\lambda} x\right) .
$$

Theorem 3.4. Let $\mathrm{E}$ be a uniformly convex and smooth Banach space and let $\mathrm{J}$ be the duality mapping on $\mathrm{E}$. Let $\mathrm{H}$ be a Hilbert space and let $\mathrm{C}$ be a nonempty closed convex subset of $\mathrm{H}$. Let $\mathrm{A}$ and $\mathrm{B}$ be maximal monotone operators of $\mathrm{H}$ into $2^{\mathrm{H}}$ such that $\operatorname{dom}(\mathrm{A}) \subset \mathrm{C}$ and $\mathrm{E}$ into $2^{\mathrm{E}^{*}}$, respectively. Let $\mathrm{J}_{\lambda}$ be the resolvent of $\mathrm{A}$ for $\lambda>0$ and let $Q_{\mu}$ be the metric resolvent of $\mathrm{B}$ for $\mu>0$. Let $\mathrm{S}: \mathrm{H} \rightarrow \mathrm{E}$ be a bounded linear operator such that $\mathrm{S} \neq 0$ and let $\mathrm{S}^{*}$ be the adjoint operator of $\mathrm{S}$. Let $\mathrm{T}: \mathrm{C} \rightarrow \mathrm{C}$ be a uniformly L-Lipschitz, uniformly asymptotically regular and asymptotically pseudo-contractive mapping in the intermediate sense with sequences $\left\{k_{n}\right\} \subset[1, \infty)$ and $\left\{v_{n}\right\} \subset[0, \infty)$ as defined in (1.4). Assume $\Gamma:=\operatorname{Fix}(T) \cap A^{-1} 0 \cap S^{-1}\left(B^{-1} 0\right)$ is nonempty and the element $x_{0} \in C$ is fixed. Let $\left\{x_{n}\right\}$ be a sequence generated by

$$
\left\{\begin{array}{l}
x_{1}, \text { chosen arbitrarily, } \\
z_{n}=J_{\lambda_{n}}\left(x_{n}-\delta_{n} S^{*} J\left(S x_{n}-Q_{\mu_{n}} S x_{n}\right)\right) \\
y_{n}=\left(1-\gamma_{n}\right) z_{n}+\gamma_{n} T^{n} z_{n}, \\
x_{n+1}=\alpha_{n} x_{0}+\left(1-\alpha_{n}\right)\left[\left(1-\beta_{n}\right) z_{n}+\beta_{n} T^{n} y_{n}\right]
\end{array}\right.
$$

where $\left\{\alpha_{n}\right\},\left\{\beta_{n}\right\},\left\{\gamma_{n}\right\} \subset[0,1]$ and $\left\{\lambda_{n}\right\},\left\{\delta_{n}\right\},\left\{\mu_{n}\right\} \subset(0,+\infty)$ satisfy the following conditions:

(i) $\lim _{n \rightarrow \infty} \alpha_{n}=0$ and $\sum_{n=1}^{\infty} \alpha_{n}=\infty$;

(ii) $0<\liminf _{n \rightarrow \infty} \beta_{n}$ and $\beta_{n} \leqslant \gamma_{n}$;

(iii) $\mathrm{a} \leqslant \gamma_{\mathrm{n}} \leqslant \mathrm{b}$ for some $\mathrm{a}>0$ and $\mathrm{b} \in\left(0, \frac{1}{\sqrt{\mathrm{k}_{\mathrm{n}}^{2}+\mathrm{L}^{2}}+\mathrm{k}_{\mathrm{n}}}\right)$;

(iv) $\sum_{n=1}^{\infty} v_{n}<\infty, v_{n}=o\left(\alpha_{n}\right), k_{n}-1=o\left(\alpha_{n}\right)$ and $\sum_{n=1}^{\infty}\left(k_{n}-1\right)<\infty$;

(v) $0<\liminf _{n \rightarrow \infty} \delta_{n} \leqslant \limsup _{n \rightarrow \infty} \delta_{n}<\frac{2}{\|S\|^{2}}, 0<\liminf _{n \rightarrow \infty} \lambda_{n} \leqslant \limsup _{n \rightarrow \infty} \lambda_{n}<\infty$ and $0<$ $\liminf _{n \rightarrow \infty} \mu_{n} \leqslant \limsup _{n \rightarrow \infty} \mu_{n}<\infty$.

Then the sequence $\left\{x_{n}\right\}$ generated by (3.3) converges strongly to a point $z_{0} \in \Gamma$, where $z_{0}=\mathrm{P}_{\Gamma} x_{0}$.

Proof. Let $u \in \Gamma$. Then we get from (3.3) that

$$
\begin{aligned}
\left\|x_{n+1}-\mathfrak{u}\right\|^{2} & =\left\|\alpha_{n}\left(x_{0}-\mathfrak{u}\right)+\left(1-\alpha_{n}\right)\left[\left(1-\beta_{n}\right)\left(z_{n}-\mathfrak{u}\right)+\beta_{n}\left(T^{n} y_{n}-u\right)\right]\right\|^{2} \\
& \leqslant \alpha_{n}\left\|x_{0}-u\right\|^{2}+\left(1-\alpha_{n}\right)\left\|\left(1-\beta_{n}\right)\left(z_{n}-u\right)+\beta_{n}\left(T^{n} y_{n}-u\right)\right\|^{2} .
\end{aligned}
$$


By Lemma 2.1, we get

$$
\begin{aligned}
& \left\|\left(1-\beta_{n}\right)\left(z_{n}-u\right)+\beta_{n}\left(T^{n} y_{n}-u\right)\right\|^{2} \\
& =\left(1-\beta_{n}\right)\left\|z_{n}-u\right\|^{2}+\beta_{n}\left\|T^{n} y_{n}-u\right\|^{2}-\beta_{n}\left(1-\beta_{n}\right)\left\|z_{n}-T^{n} y_{n}\right\|^{2} .
\end{aligned}
$$

Choose $y=u$ in (1.5) to derive

$$
\left\|T^{n} x-u\right\|^{2} \leqslant\left(2 k_{n}-1\right)\|x-u\|^{2}+\left\|x-T^{n} x\right\|^{2}+2 v_{n} .
$$

From (3.5) and Lemma 2.1, we obtain

$$
\begin{aligned}
\left\|T^{n} y_{n}-u\right\|^{2} \leqslant & \left(2 k_{n}-1\right)\left\|y_{n}-u\right\|^{2}+\left\|y_{n}-T^{n} y_{n}\right\|^{2}+2 v_{n} \\
= & \left(2 k_{n}-1\right)\left\|\left(1-\gamma_{n}\right) z_{n}+\gamma_{n} T^{n} z_{n}-u\right\|^{2}+\left\|\left(1-\gamma_{n}\right) z_{n}+\gamma_{n} T^{n} z_{n}-T^{n} y_{n}\right\|^{2}+2 v_{n} \\
= & \left(2 k_{n}-1\right)\left\|\left(1-\gamma_{n}\right)\left(z_{n}-u\right)+\gamma_{n}\left(T^{n} z_{n}-u\right)\right\|^{2} \\
& +\left\|\left(1-\gamma_{n}\right)\left(z_{n}-T^{n} y_{n}\right)+\gamma_{n}\left(T^{n} z_{n}-T^{n} y_{n}\right)\right\|^{2}+2 v_{n} \\
= & \left(2 k_{n}-1\right)\left[\left(1-\gamma_{n}\right)\left\|z_{n}-u\right\|^{2}+\gamma_{n}\left\|T^{n} z_{n}-u\right\|^{2}-\gamma_{n}\left(1-\gamma_{n}\right)\left\|z_{n}-T^{n} z_{n}\right\|^{2}\right] \\
& +\left(1-\gamma_{n}\right)\left\|z_{n}-T^{n} y_{n}\right\|^{2}+\gamma_{n}\left\|T^{n} z_{n}-T^{n} y_{n}\right\|^{2}-\gamma_{n}\left(1-\gamma_{n}\right)\left\|T^{n} z_{n}-z_{n}\right\|^{2}+2 v_{n} \\
= & \left(2 k_{n}-1\right)\left[\left(1-\gamma_{n}\right)\left\|z_{n}-u\right\|^{2}+\gamma_{n}\left(2 k_{n}-1\right)\left\|z_{n}-u\right\|^{2}+\gamma_{n}\left\|z_{n}-T^{n} z_{n}\right\|^{2}+2 \gamma_{n} v_{n}\right. \\
& \left.-\gamma_{n}\left(1-\gamma_{n}\right)\left\|z_{n}-T^{n} z_{n}\right\|^{2}\right]+\left(1-\gamma_{n}\right)\left\|z_{n}-T^{n} y_{n}\right\|^{2}+\gamma_{n}\left\|T^{n} z_{n}-T^{n} y_{n}\right\|^{2} \\
& -\gamma_{n}\left(1-\gamma_{n}\right)\left\|T^{n} z_{n}-z_{n}\right\|^{2}+2 v_{n} \\
= & \left(2 k_{n}-1\right)\left[\left(1-\gamma_{n}\right)\left\|z_{n}-u\right\|^{2}+\gamma_{n}\left(2 k_{n}-1\right)\left\|z_{n}-u\right\|^{2}+2 \gamma_{n} v_{n}+\gamma_{n}^{2}\left\|z_{n}-T^{n} z_{n}\right\|^{2}\right] \\
& +\left(1-\gamma_{n}\right)\left\|z_{n}-T^{n} y_{n}\right\|^{2}+\gamma_{n}\left\|T^{n} z_{n}-T^{n} y_{n}\right\|^{2}-\gamma_{n}\left(1-\gamma_{n}\right)\left\|T^{n} z_{n}-z_{n}\right\|^{2}+2 v_{n} .
\end{aligned}
$$

Observe that

$$
\left\|z_{n}-y_{n}\right\|=\gamma_{n}\left\|z_{n}-T^{n} z_{n}\right\|
$$

Since T is uniformly L-Lipschitz, from (3.6) and (3.7), we deduce

$$
\begin{aligned}
\left\|T^{n} y_{n}-u\right\|^{2} \leqslant & \left(2 k_{n}-1\right)\left[\left(1-\gamma_{n}\right)\left\|z_{n}-u\right\|^{2}+\gamma_{n}\left(2 k_{n}-1\right)\left\|z_{n}-u\right\|^{2}+2 \gamma_{n} v_{n}+\gamma_{n}^{2}\left\|z_{n}-T^{n} z_{n}\right\|^{2}\right] \\
& +\left(1-\gamma_{n}\right)\left\|z_{n}-T^{n} y_{n}\right\|^{2}+\gamma_{n}^{3} L^{2}\left\|z_{n}-T^{n} z_{n}\right\|^{2}-\gamma_{n}\left(1-\gamma_{n}\right)\left\|T^{n} z_{n}-z_{n}\right\|^{2}+2 v_{n} \\
= & {\left[\left(2 k_{n}-1\right)\left(1-\gamma_{n}\right)+\left(2 k_{n}-1\right)^{2} \gamma_{n}\right]\left\|z_{n}-u\right\|^{2} } \\
& +\left[\left(2 k_{n}-1\right) \gamma_{n}^{2}+\gamma_{n}^{3} L^{2}-\gamma_{n}\left(1-\gamma_{n}\right)\right]\left\|z_{n}-T^{n} z_{n}\right\|^{2}+2\left(2 k_{n}-1\right) \gamma_{n} v_{n} \\
& +\left(1-\gamma_{n}\right)\left\|z_{n}-T^{n} y_{n}\right\|^{2}+2 v_{n} \\
= & {\left[1+2\left(2 k_{n} \gamma_{n}-\gamma_{n}+1\right)\left(k_{n}-1\right)\right]\left\|z_{n}-u\right\|^{2}-\gamma_{n}\left(1-2 k_{n} \gamma_{n}-\gamma_{n}^{2} L^{2}\right)\left\|z_{n}-T^{n} z_{n}\right\|^{2} } \\
& +2\left(2 k_{n}-1\right) \gamma_{n} v_{n}+\left(1-\gamma_{n}\right)\left\|z_{n}-T^{n} y_{n}\right\|^{2}+2 v_{n} .
\end{aligned}
$$

By condition (iii), we know that $\gamma_{n} \leqslant b<\frac{1}{\sqrt{k_{n}^{2}+L^{2}}+k_{n}}$ for all $n \geqslant 1$. Then, we deduce that $1-2 k_{n} \gamma_{n}-$ $\gamma_{n}^{2} \mathrm{~L}^{2}>0$ for all $\mathrm{n} \geqslant 1$. Thus, we have from (3.8) that

$$
\left\|T^{n} y_{n}-u\right\|^{2} \leqslant\left[1+2\left(2 k_{n} \gamma_{n}-\gamma_{n}+1\right)\left(k_{n}-1\right)\right]\left\|z_{n}-u\right\|^{2}+4 k_{n} \nu_{n}+\left(1-\gamma_{n}\right)\left\|z_{n}-T^{n} y_{n}\right\|^{2} .
$$

It follows from (3.4), (3.9), Lemma 3.3, and condition (ii) that

$$
\begin{aligned}
& \left\|\left(1-\beta_{n}\right)\left(z_{n}-u\right)+\beta_{n}\left(T^{n} y_{n}-u\right)\right\|^{2} \\
& \leqslant\left(1-\beta_{n}\right)\left\|z_{n}-u\right\|^{2}+\beta_{n}\left[1+2\left(2 k_{n} \gamma_{n}-\gamma_{n}+1\right)\left(k_{n}-1\right)\right]\left\|z_{n}-u\right\|^{2} \\
& \quad+4 \beta_{n} k_{n} v_{n}+\beta_{n}\left(1-\gamma_{n}\right)\left\|z_{n}-T^{n} y_{n}\right\|^{2}-\beta_{n}\left(1-\beta_{n}\right)\left\|z_{n}-T^{n} y_{n}\right\|^{2}
\end{aligned}
$$




$$
\begin{aligned}
& \leqslant\left[1+2 \beta_{n}\left(2 k_{n} \gamma_{n}-\gamma_{n}+1\right)\left(k_{n}-1\right)\right]\left\|z_{n}-u\right\|^{2}+\beta_{n}\left(\beta_{n}-\gamma_{n}\right)\left\|z_{n}-T^{n} y_{n}\right\|^{2}+4 \beta_{n} k_{n} v_{n} \\
& \leqslant\left[1+2\left(2 k_{n} \gamma_{n}-\gamma_{n}+1\right)\left(k_{n}-1\right)\right]\left\|x_{n}-u\right\|^{2}+4 k_{n} v_{n} .
\end{aligned}
$$

Thus, we get that

$$
\begin{aligned}
\left\|x_{n+1}-u\right\|^{2} & =\left\|\alpha_{n} x_{0}+\left(1-\alpha_{n}\right)\left[\left(1-\beta_{n}\right) z_{n}+\beta_{n} T^{n} y_{n}\right]-u\right\|^{2} \\
& \leqslant \alpha_{n}\left\|x_{0}-u\right\|^{2}+\left(1-\alpha_{n}\right)\left\|\left(1-\beta_{n}\right)\left(z_{n}-u\right)+\beta_{n}\left(T^{n} y_{n}-u\right)\right\|^{2} \\
& \leqslant \max \left\{\left\|x_{0}-u\right\|^{2},\left[1+2\left(2 k_{n} \gamma_{n}-\gamma_{n}+1\right)\left(k_{n}-1\right)\right]\left\|x_{n}-u\right\|^{2}+4 \beta_{n} k_{n} v_{n}\right\} \\
& \leqslant \max \left\{\left\|x_{0}-u\right\|^{2},\left[1+M_{1}\left(k_{n}-1\right)\right]\left\|x_{n}-u\right\|^{2}+M_{2} v_{n}\right\},
\end{aligned}
$$

where $M_{1}=\sup _{n \geqslant 1}\left\{2\left(2 k_{n} \gamma_{n}-\gamma_{n}+1\right)\right\}$ and $M_{2}=\sup _{n \geqslant 1}\left\{4 k_{n}\right\}$. This implies that the sequence $\left\{x_{n}\right\}$ is bounded by Lemma 2.4. In terms of Lemma 2.2 and (3.3), we have

$$
\begin{aligned}
\left\|x_{n+1}-u\right\|^{2}= & \left\|\alpha_{n}\left(x_{0}-u\right)+\left(1-\alpha_{n}\right)\left(z_{n}-u\right)+\left(1-\alpha_{n}\right) \beta_{n}\left(T^{n} y_{n}-x_{n}\right)\right\|^{2} \\
\leqslant & \left\|\left(1-\alpha_{n}\right)\left(z_{n}-u\right)+\left(1-\alpha_{n}\right) \beta_{n}\left(T^{n} y_{n}-z_{n}\right)\right\|^{2}+2 \alpha_{n}\left\langle x_{0}-u, x_{n+1}-u\right\rangle \\
= & \left(1-\alpha_{n}\right)^{2}\left\|z_{n}-u\right\|^{2}+\left(1-\alpha_{n}\right)^{2} \beta_{n}^{2}\left\|T^{n} y_{n}-z_{n}\right\|^{2} \\
& +2\left(1-\alpha_{n}\right)^{2} \beta_{n}\left\langle z_{n}-u, T^{n} y_{n}-z_{n}\right\rangle+2 \alpha_{n}\left\langle x_{0}-u, x_{n+1}-u\right\rangle .
\end{aligned}
$$

Combining Lemma 2.1 and (3.8) yields

$$
\begin{aligned}
& 2\left\langle z_{n}-u, T^{n} y_{n}-z_{n}\right\rangle \\
&=\left\|T^{n} y_{n}-u\right\|^{2}-\left\|T^{n} y_{n}-z_{n}\right\|^{2}-\left\|z_{n}-u\right\|^{2} \\
& \leqslant {\left[1+2\left(2 k_{n} \gamma_{n}-\gamma_{n}+1\right)\left(k_{n}-1\right)\right]\left\|z_{n}-u\right\|^{2}-\gamma_{n}\left(1-2 k_{n} \gamma_{n}-\gamma_{n}^{2} L^{2}\right)\left\|z_{n}-T^{n} z_{n}\right\|^{2} } \\
&+2\left(2 k_{n}-1\right) \gamma_{n} v_{n}+\left(1-\gamma_{n}\right)\left\|z_{n}-T^{n} y_{n}\right\|^{2}+2 v_{n}-\left\|T^{n} y_{n}-z_{n}\right\|^{2}-\left\|z_{n}-u\right\|^{2} \\
& \leqslant 2\left(2 k_{n} \gamma_{n}-\gamma_{n}+1\right)\left(k_{n}-1\right)\left\|z_{n}-u\right\|^{2}-\gamma_{n}\left(1-2 k_{n} \gamma_{n}-\gamma_{n}^{2} L^{2}\right)\left\|z_{n}-T^{n} z_{n}\right\|^{2} \\
&-\gamma_{n}\left\|z_{n}-T^{n} y_{n}\right\|^{2}+4 k_{n} v_{n} .
\end{aligned}
$$

Now put $J_{\lambda_{n}}=\frac{1}{2}\left(I+K_{n}\right)$ for all $n \geqslant 1$. Since $J_{\lambda_{n}}$ is firmly nonexpansive, then we know that $K_{n}$ is nonexpansive and $\operatorname{Fix}\left(\mathrm{J}_{\lambda_{n}}\right)=\operatorname{Fix}\left(\mathrm{K}_{n}\right)$ for all $n \geqslant 1$. Therefor, we get

$$
\begin{aligned}
\left\|z_{n}-u\right\|^{2}= & \left\|J_{\lambda_{n}}\left(x_{n}-\delta_{n} S^{*} J\left(S x_{n}-Q_{\mu_{n}} S x_{n}\right)\right)-u\right\|^{2} \\
= & \frac{1}{2}\left\|\left(x_{n}-\delta_{n} S^{*} J\left(S x_{n}-Q_{\mu_{n}} S x_{n}\right)\right)-u\right\|^{2}+\frac{1}{2}\left\|K_{n}\left(x_{n}-\delta_{n} S^{*} J\left(S x_{n}-Q_{\mu_{n}} S x_{n}\right)\right)-u\right\|^{2} \\
& -\frac{1}{4}\left\|K_{n}\left(x_{n}-\delta_{n} S^{*} J\left(S x_{n}-Q_{\mu_{n}} S x_{n}\right)\right)-\left(x_{n}-\delta_{n} S^{*} J\left(S x_{n}-Q_{\mu_{n}} S x_{n}\right)\right)\right\|^{2} \\
\leqslant & \left\|x_{n}-\delta_{n} S^{*} J\left(S x_{n}-Q_{\mu_{n}} S x_{n}\right)-u\right\|^{2} \\
& -\frac{1}{4}\left\|K_{n}\left(x_{n}-\delta_{n} S^{*} J\left(S x_{n}-Q_{\mu_{n}} S x_{n}\right)\right)-\left(x_{n}-\delta_{n} S^{*} J\left(S x_{n}-Q_{\mu_{n}} S x_{n}\right)\right)\right\|^{2} \\
\leqslant & \left\|x_{n}-u\right\|^{2}+\left\|\delta_{n} S^{*} J\left(S x_{n}-Q_{\mu_{n}} S x_{n}\right)\right\|^{2}-2 \delta_{n}\left\langle x_{n}-u, S^{*} J\left(S x_{n}-Q_{\mu_{n}} S x_{n}\right)\right\rangle \\
& -\frac{1}{4}\left\|K_{n}\left(x_{n}-\delta_{n} S^{*} J\left(S x_{n}-Q_{\mu_{n}} S x_{n}\right)\right)-\left(x_{n}-\delta_{n} S^{*} J\left(S x_{n}-Q_{\mu_{n}} S x_{n}\right)\right)\right\|^{2} \\
\leqslant & \left\|x_{n}-u\right\|^{2}+\left\|\delta_{n} S^{*} J\left(S x_{n}-Q_{\mu_{n}} S x_{n}\right)\right\|^{2} \\
& -2 \delta_{n}\left\langle S x_{n}-Q_{\mu_{n}} S x_{n}+Q_{\mu_{n}} S x_{n}-S u, J\left(S x_{n}-Q_{\mu_{n}} S x_{n}\right)\right\rangle \\
& -\frac{1}{4}\left\|K_{n}\left(x_{n}-\delta_{n} S^{*} J\left(S x_{n}-Q_{\mu_{n}} S x_{n}\right)\right)-\left(x_{n}-\delta_{n} S^{*} J\left(S x_{n}-Q_{\mu_{n}} S x_{n}\right)\right)\right\|^{2} \\
\leqslant & \left\|x_{n}-u\right\|^{2}+\delta_{n}^{2}\|S\|^{2}\left\|S x_{n}-Q_{\mu_{n}} S x_{n}\right\|^{2}
\end{aligned}
$$




$$
\begin{aligned}
& -2 \delta_{n}\left\langle Q_{\mu_{n}} S x_{n}-S u, J\left(S x_{n}-Q_{\mu_{n}} S x_{n}\right)\right\rangle-2 \delta_{n}\left\|S x_{n}-Q_{\mu_{n}} S x_{n}\right\|^{2} \\
- & \frac{1}{4}\left\|K_{n}\left(x_{n}-\delta_{n} S^{*} J\left(S x_{n}-Q_{\mu_{n}} S x_{n}\right)\right)-\left(x_{n}-\delta_{n} S^{*} J\left(S x_{n}-Q_{\mu_{n}} S x_{n}\right)\right)\right\|^{2} \\
\leqslant & \left\|x_{n}-u\right\|^{2}+\delta_{n}^{2}\|S\|^{2}\left\|S x_{n}-Q_{\mu_{n}} S x_{n}\right\|^{2}-2 \delta_{n}\left\|S x_{n}-Q_{\mu_{n}} S x_{n}\right\|^{2} \\
& -\frac{1}{4}\left\|K_{n}\left(x_{n}-\delta_{n} S^{*} J\left(S x_{n}-Q_{\mu_{n}} S x_{n}\right)\right)-\left(x_{n}-\delta_{n} S^{*} J\left(S x_{n}-Q_{\mu_{n}} S x_{n}\right)\right)\right\|^{2} \\
= & \left\|x_{n}-u\right\|^{2}-\delta_{n}\left(2-\delta_{n}\|S\|^{2}\right)\left\|S x_{n}-Q_{\mu_{n}} S x_{n}\right\|^{2} \\
& -\frac{1}{4}\left\|K_{n}\left(x_{n}-\delta_{n} S^{*} J\left(S x_{n}-Q_{\mu_{n}} S x_{n}\right)\right)-\left(x_{n}-\delta_{n} S^{*} J\left(S x_{n}-Q_{\mu_{n}} S x_{n}\right)\right)\right\|^{2} .
\end{aligned}
$$

It follows from (3.10), (3.11), and (3.12) that

$$
\begin{aligned}
\left\|x_{n+1}-u\right\|^{2} \leqslant & \left(1-\alpha_{n}\right)^{2}\left\|z_{n}-u\right\|^{2}+\left(1-\alpha_{n}\right)^{2} \beta_{n}^{2}\left\|T^{n} y_{n}-z_{n}\right\|^{2} \\
& +2\left(1-\alpha_{n}\right)^{2} \beta_{n}\left(2 k_{n} \gamma_{n}-\gamma_{n}+1\right)\left(k_{n}-1\right)\left\|z_{n}-u\right\|^{2} \\
& -\left(1-\alpha_{n}\right)^{2} \beta_{n} \gamma_{n}\left(1-2 k_{n} \gamma_{n}-\gamma_{n}^{2} L^{2}\right)\left\|z_{n}-T^{n} z_{n}\right\|^{2} \\
& -\left(1-\alpha_{n}\right)^{2} \beta_{n} \gamma_{n}\left\|T^{n} y_{n}-z_{n}\right\|^{2} \\
& +4\left(1-\alpha_{n}\right)^{2} \beta_{n} k_{n} v_{n}+2 \alpha_{n}\left\langle x_{0}-u, x_{n+1}-u\right\rangle \\
\leqslant & \left(1-\alpha_{n}\right)\left\|z_{n}-u\right\|^{2}+2\left(1-\alpha_{n}\right)^{2} \beta_{n}\left(2 k_{n} \gamma_{n}-\gamma_{n}+1\right)\left(k_{n}-1\right)\left\|z_{n}-u\right\|^{2} \\
& -\left(1-\alpha_{n}\right)^{2} \beta_{n} \gamma_{n}\left(1-2 k_{n} \gamma_{n}-\gamma_{n}^{2} L^{2}\right)\left\|z_{n}-T^{n} z_{n}\right\|^{2} \\
& +4\left(1-\alpha_{n}\right)^{2} \beta_{n} k_{n} v_{n}+2 \alpha_{n}\left\langle x_{0}-u, x_{n+1}-u\right\rangle \\
\leqslant & \left(1-\alpha_{n}\right)\left\|z_{n}-u\right\|^{2}+2\left(2 k_{n} \gamma_{n}-\gamma_{n}+1\right)\left(k_{n}-1\right)\left\|z_{n}-u\right\|^{2} \\
& -\left(1-\alpha_{n}\right)^{2} \beta_{n} \gamma_{n}\left(1-2 k_{n} \gamma_{n}-\gamma_{n}^{2} L^{2}\right)\left\|z_{n}-T^{n} z_{n}\right\|^{2}+4 k_{n} v_{n} \\
& +2 \alpha_{n}\left\langle x_{0}-u, x_{n}-u\right\rangle \\
\leqslant & \left(1-\alpha_{n}\right)\left\|x_{n}-u\right\|^{2}-\left(1-\alpha_{n}\right) \delta_{n}\left(2-\delta_{n}\|S\|^{2}\right)\left\|S x_{n}-Q_{\mu_{n}} S x_{n}\right\|^{2} \\
& -\frac{1}{4}\left(1-\alpha_{n}\right)\left\|k_{n}\left(x_{n}-\delta_{n} S^{*} J\left(S x_{n}-Q \mu_{n} S x_{n}\right)\right)-\left(x_{n}-\delta_{n} S^{*} J\left(S x_{n}-Q_{\mu_{n}} S x_{n}\right)\right)\right\|^{2} \\
& +M_{1}\left(k_{n}-1\right)\left\|x_{n}-u\right\|^{2}+2 \alpha_{n}\left\langle x_{0}-u, x_{n+1}-u\right\rangle \\
& -\left(1-\alpha_{n}\right)^{2} \beta_{n} \gamma_{n}\left(1-2 k_{n} \gamma_{n}-\gamma_{n}^{2} L^{2}\right)\left\|z_{n}-T^{n} z_{n}\right\|^{2}+M_{2} v_{n},
\end{aligned}
$$

which implies that

$$
\begin{aligned}
(1 & \left.-\alpha_{n}\right) \delta_{n}\left(2-\delta_{n}\|S\|^{2}\right)\left\|S x_{n}-Q_{\mu_{n}} S x_{n}\right\|^{2} \\
& +\frac{1}{4}\left(1-\alpha_{n}\right)\left\|K_{n}\left(x_{n}-\delta_{n} S^{*} J\left(S x_{n}-Q_{\mu_{n}} S x_{n}\right)\right)-\left(x_{n}-\delta_{n} S^{*} J\left(S x_{n}-Q_{\mu_{n}} S x_{n}\right)\right)\right\|^{2} \\
& +\left(1-\alpha_{n}\right)^{2} \beta_{n} \gamma_{n}\left(1-2 k_{n} \gamma_{n}-\gamma_{n}^{2} L^{2}\right)\left\|z_{n}-T^{n} z_{n}\right\|^{2} \\
\leqslant & \left\|x_{n}-u\right\|^{2}-\left\|x_{n+1}-u\right\|^{2}+M_{1}\left(k_{n}-1\right)\left\|x_{n}-u\right\|^{2}+M_{2} v_{n} \\
& +2 \alpha_{n}\left\langle x_{0}-u, x_{n+1}-u\right\rangle .
\end{aligned}
$$

Case 1. Assume there exists some integer $m>0$ such that $\left\{\left\|x_{n}-u\right\|\right\}$ is decreasing for all $n \geqslant m$. In this case, we know that $\lim _{n \rightarrow \infty}\left\|x_{n}-u\right\|$ exists. From (3.14), conditions (i)-(v), we deduce

$$
\begin{array}{r}
\lim _{n \rightarrow \infty}\left\|z_{n}-T^{n} z_{n}\right\|=0, \\
\lim _{n \rightarrow \infty}\left\|K_{n}\left(x_{n}-\delta_{n} S^{*} J\left(S x_{n}-Q_{\mu_{n}} S x_{n}\right)\right)-\left(x_{n}-\delta_{n} S^{*} J\left(S x_{n}-Q_{\mu_{n}} S x_{n}\right)\right)\right\|=0
\end{array}
$$

and

$$
\lim _{n \rightarrow \infty}\left\|S x_{n}-Q_{\mu_{n}} S x_{n}\right\|=0 .
$$


It follows from (3.16) and (3.17) that

$$
\begin{aligned}
\left\|z_{n}-x_{n}\right\|= & \left\|J_{\lambda_{n}}\left(x_{n}-\delta_{n} S^{*} J\left(S x_{n}-Q_{\mu_{n}} S x_{n}\right)\right)-x_{n}\right\| \\
= & \left\|\frac{1}{2}\left(x_{n}-\delta_{n} S^{*} J\left(S x_{n}-Q_{\mu_{n}} S x_{n}\right)\right)+\frac{1}{2} K_{n}\left(x_{n}-\delta_{n} S^{*} J\left(S x_{n}-Q_{\mu_{n}} S x_{n}\right)\right)-x_{n}\right\| \\
\leqslant & \frac{1}{2}\left\|\delta_{n} S^{*} J\left(S x_{n}-Q_{\mu_{n}} S x_{n}\right)\right\|+\frac{1}{2}\left\|K_{n}\left(x_{n}-\delta_{n} S^{*} J\left(S x_{n}-Q_{\mu_{n}} S x_{n}\right)\right)-x_{n}\right\| \\
\leqslant & \frac{1}{2}\left\|\delta_{n} S^{*} J\left(S x_{n}-Q_{\mu_{n}} S x_{n}\right)\right\| \\
& +\frac{1}{2}\left\|K_{n}\left(x_{n}-\delta_{n} S^{*} J\left(S x_{n}-Q_{\mu_{n}} S x_{n}\right)\right)-\left(x_{n}-\delta_{n} S^{*} J\left(S x_{n}-Q_{\mu_{n}} S x_{n}\right)\right)\right\| \\
& +\frac{1}{2}\left\|\left(x_{n}-\delta_{n} S^{*} J\left(S x_{n}-Q_{\mu_{n}} S x_{n}\right)\right)-x_{n}\right\| \\
\leqslant & \lambda_{n}\|S\|\left\|S x_{n}-Q_{\mu_{n}} S x_{n}\right\| \\
& +\frac{1}{2}\left\|K_{n}\left(x_{n}-\delta_{n} S^{*} J\left(S x_{n}-Q_{\mu_{n}} S x_{n}\right)\right)-\left(x_{n}-\delta_{n} S^{*} J\left(S x_{n}-Q_{\mu_{n}} S x_{n}\right)\right)\right\| \rightarrow 0 .
\end{aligned}
$$

Observe that

$$
\begin{aligned}
\left\|J_{\lambda_{n}} x_{n}-x_{n}\right\| & \leqslant\left\|J_{\lambda_{n}} x_{n}-z_{n}\right\|+\left\|z_{n}-x_{n}\right\| \\
& \leqslant\left\|\delta_{n} S^{*} J\left(S x_{n}-Q_{\mu_{n}} S x_{n}\right)\right\|+\left\|z_{n}-x_{n}\right\| \\
& \leqslant \lambda_{n}\|S\|\left\|S x_{n}-Q_{\mu_{n}} S x_{n}\right\|+\left\|z_{n}-x_{n}\right\| .
\end{aligned}
$$

This together with (3.17) and (3.18) implies

$$
\lim _{n \rightarrow \infty}\left\|J_{\lambda_{n}} x_{n}-x_{n}\right\|=0
$$

It follows from (3.7) and (3.15) that

$$
\lim _{n \rightarrow \infty}\left\|z_{n}-y_{n}\right\|=0
$$

Since $T$ is uniformly L-Lipschitz, we have

$$
\lim _{n \rightarrow \infty}\left\|T^{n} z_{n}-T^{n} y_{n}\right\| \leqslant L \lim _{n \rightarrow \infty}\left\|z_{n}-y_{n}\right\|=0 .
$$

Taking into consideration that

$$
\left\|x_{n}-T^{n} y_{n}\right\| \leqslant\left\|x_{n}-z_{n}\right\|+\left\|z_{n}-T^{n} z_{n}\right\|+\left\|T^{n} z_{n}-T^{n} y_{n}\right\|,
$$

we deduce from (3.15), (3.18), (3.20), and (3.21) that

$$
\lim _{n \rightarrow \infty}\left\|x_{n}-T^{n} y_{n}\right\|=0
$$

In view of (3.3), we get

$$
\left\|x_{n+1}-x_{n}\right\| \leqslant \alpha_{n}\left\|x_{0}-x_{n}\right\|+\left(1-\alpha_{n}\right)\left(1-\beta_{n}\right)\left\|z_{n}-x_{n}\right\|+\left(1-\alpha_{n}\right) \beta_{n}\left\|T^{n} y_{n}-x_{n}\right\| .
$$

This together with (3.18), (3.22), and condition (i) implies that

$$
\lim _{n \rightarrow \infty}\left\|x_{n+1}-x_{n}\right\|=0 \text {. }
$$

It is clear that

$$
\left\|x_{n}-T^{n} x_{n}\right\| \leqslant\left\|x_{n}-z_{n}\right\|+\left\|z_{n}-T^{n} z_{n}\right\|+\left\|T^{n} z_{n}-T^{n} x_{n}\right\|
$$


Again since T is uniformly L-Lipschitz, then we derive from (3.15), (3.18), and (3.24) that

$$
\lim _{n \rightarrow \infty}\left\|x_{n}-T^{n} x_{n}\right\|=0 .
$$

Notice

$$
\begin{aligned}
\left\|x_{n+1}-T x_{n+1}\right\| & \leqslant\left\|x_{n+1}-T^{n+1} x_{n+1}\right\|+\left\|T^{n+1} x_{n+1}-T^{n+1} x_{n}\right\|+\left\|T^{n+1} x_{n}-T x_{n+1}\right\| \\
& \leqslant\left\|x_{n+1}-T^{n+1} x_{n+1}\right\|+L\left\|x_{n+1}-x_{n}\right\|+L\left\|T^{n} x_{n}-x_{n+1}\right\| \\
& \leqslant\left\|x_{n+1}-T^{n+1} x_{n+1}\right\|+2 L\left\|x_{n+1}-x_{n}\right\|+L\left\|T^{n} x_{n}-x_{n}\right\| .
\end{aligned}
$$

By (3.23), (3.25), and (3.26), we conclude that

$$
\lim _{n \rightarrow \infty}\left\|x_{n+1}-T x_{n+1}\right\|=0 .
$$

Since $\left\{x_{n}\right\}$ is bounded, there exists a subsequence $\left\{x_{n_{i}}\right\}$ of $\left\{x_{n}\right\}$ satisfying $x_{n_{i}} \rightarrow \tilde{x} \in C$. Without loss of generality, we may also assume

$$
\lim _{i \rightarrow \infty}\left\langle x_{0}-z_{0}, x_{n_{i}}-z_{0}\right\rangle=\limsup _{n \rightarrow \infty}\left\langle x_{0}-z_{0}, x_{n}-z_{0}\right\rangle .
$$

Since $S$ is bounded and linear, we also see that $S x_{n_{i}} \rightarrow S \tilde{x}$ as $i \rightarrow \infty$. Noticing (3.17), we also have $Q_{\mu_{n_{i}}} S x_{n_{i}} \rightarrow S \tilde{x}$ as $i \rightarrow \infty$. Since $Q_{\mu_{n}}$ is the metric resolvent of $B$ for $\mu_{n}>0$, then we obtain $\frac{J\left(S x_{n}-Q_{\mu_{n}} S x_{n}\right)}{\mu_{n}} \in$ $B Q_{\mu_{n}} S x_{n}$ for all $n \in N$. From the monotonicity of $B$ we see that

$$
0 \leqslant\left\langle u-Q_{\mu_{n_{i}}} S x_{n_{i}}, \tilde{u}-\frac{J\left(S x_{n_{i}}-Q_{\mu_{n_{i}}} S x_{n_{i}}\right)}{\mu_{n_{i}}}\right\rangle, \quad \forall(u, \tilde{u}) \in B .
$$

Letting $i \rightarrow \infty$ and noticing $\left\|J\left(S x_{n_{i}}-Q_{\mu_{n_{i}}} S x_{n_{i}}\right)\right\|=\left\|S x_{n_{i}}-Q_{\mu_{n_{i}}} S x_{n_{i}}\right\| \rightarrow 0$, we deduce that

$$
0 \leqslant\langle u-S \tilde{x}, \tilde{u}-0\rangle, \quad \forall(u, \tilde{u}) \in B
$$

by (3.17) and condition (v). Since $B$ is maximal monotone, we get $S \tilde{x} \in B^{-1} 0$. Hence, $\tilde{x} \in S^{-1} B^{-1} 0$. Similarly, noticing (3.19), we also get $\tilde{x} \in A^{-1} 0$. Therefor, we have $\tilde{x} \in A^{-1} 0 \bigcap S^{-1} B^{-1} 0$. Meanwhile, using Lemma 2.5 and (3.26), we obtain $\tilde{x} \in \operatorname{Fix}(T)$. And hence it follows from (3.27) and Lemma 2.6 that

$$
\limsup _{n \rightarrow \infty}\left\langle x_{0}-z_{0}, x_{n}-z_{0}\right\rangle=\lim _{i \rightarrow \infty}\left\langle x_{0}-z_{0}, x_{n_{i}}-z_{0}\right\rangle=\left\langle x_{0}-z_{0}, \tilde{x}-z_{0}\right\rangle=\left\langle x_{0}-P_{\Gamma} x_{0}, \tilde{x}-P_{\Gamma} x_{0}\right\rangle \leqslant 0 .
$$

By (3.13), we deduce

$$
\left\|x_{n+1}-z_{0}\right\|^{2} \leqslant\left(1-\alpha_{n}\right)\left\|x_{n}-z_{0}\right\|^{2}+M_{1}\left(k_{n}-1\right)\left\|x_{n}-z_{0}\right\|^{2}+M_{2} v_{n}+2 \alpha_{n}\left\langle x_{0}-z_{0}, x_{n+1}-z_{0}\right\rangle .
$$

Applying Lemma 2.7 and (3.23) to (3.28), we deduce $x_{n} \rightarrow z_{0}$.

Case 2. Suppose that there exists $\left\{n_{i}\right\}$ of $\{n\}$ such that $\left\|x_{n_{i}}-z_{0}\right\|<\left\|x_{n_{i}+1}-z_{0}\right\|$ for all $i \in \mathbb{N}$. Then by Lemma 2.3, there exists a nondecreasing sequence $\left\{m_{\mathfrak{j}}\right\}$ in $\mathbb{N}$ such that

$$
\left\|x_{m_{j}}-z_{0}\right\| \leqslant\left\|x_{m_{j}+1}-z_{0}\right\| \quad \text { and } \quad\left\|x_{j}-z_{0}\right\| \leqslant\left\|x_{m_{j}+1}-z_{0}\right\| .
$$

We want to show that

$$
\limsup _{j \rightarrow \infty}\left\langle x_{0}-z_{0}, x_{m_{j}}-z_{0}\right\rangle \leqslant 0,
$$

where $z_{0}=\mathrm{P}_{\Gamma} x_{0}$. Without loss of generality, there exists a subsequence $\left\{x_{m_{\mathfrak{j}_{k}}}\right\}$ of $\left\{x_{m_{j}}\right\}$ such that $x_{m_{j_{k}}} \rightarrow \omega$ for some $\omega \in \mathrm{C}$ and

$$
\lim _{k \rightarrow \infty}\left\langle x_{0}-z_{0}, x_{m_{j_{k}}}-z_{0}\right\rangle=\limsup _{j \rightarrow \infty}\left\langle x_{0}-z_{0}, x_{m_{j}}-z_{0}\right\rangle .
$$


Following a similar argument as in the proof of Case 1, we also have

$$
\lim _{j \rightarrow \infty}\left\|x_{m_{j}}-T^{m_{j}} x_{m_{j}}\right\|=0
$$

and

$$
\lim _{j \rightarrow \infty}\left\|x_{m_{j}+1}-x_{m_{j}}\right\|=0 .
$$

Since $T$ is uniformly asymptotically regular, we derive from (3.30) that

$$
\begin{aligned}
\left\|x_{m_{j}}-T x_{m_{j}}\right\| & \leqslant\left\|x_{m_{j}}-T^{m_{j}} x_{m_{j}}\right\|+\left\|T^{m_{j}} x_{m_{j}}-T^{m_{j}+1} x_{m_{j}}\right\|+\left\|T^{m_{j}+1} x_{m_{j}}-T x_{m_{j}}\right\| \\
& \leqslant\left\|x_{m_{j}}-T^{m_{j}} x_{m_{j}}\right\|+\left\|T^{m_{j}} x_{m_{j}}-T^{m_{j}+1} x_{m_{j}}\right\|+L\left\|T^{m_{j}} x_{m_{j}}-x_{m_{j}}\right\| \\
& \leqslant(L+1)\left\|x_{m_{j}}-T^{m_{j}} x_{m_{j}}\right\|+\left\|T^{m_{j}} x_{m_{j}}-T^{m_{j}+1} x_{m_{j}}\right\| \rightarrow 0 .
\end{aligned}
$$

By virtue of Lemma 2.5 and (3.32), we deduce that $\omega \in \operatorname{Fix}(T)$. Like in Case 1, we can also obtain $\omega \in A^{-1} 0 \bigcap S^{-1}\left(B^{-1} 0\right)$. Thus we obtain

$$
\limsup _{j \rightarrow \infty}\left\langle x_{0}-z_{0}, x_{m_{j}}-z_{0}\right\rangle=\lim _{k \rightarrow \infty}\left\langle x_{0}-z_{0}, x_{m_{j_{k}}}-z_{0}\right\rangle=\left\langle x_{0}-P_{\Gamma} x_{0}, \omega-P_{\Gamma} x_{0}\right\rangle \leqslant 0 .
$$

Since $\left\|x_{m_{j}}-z_{0}\right\| \leqslant\left\|x_{m_{j}+1}-z_{0}\right\|$, we deduce from (3.13) that

$$
\begin{aligned}
\left\|x_{m_{j}}-z_{0}\right\|^{2} \leqslant & \left\|x_{m_{j}+1}-z_{0}\right\|^{2} \\
\leqslant & \left(1-\alpha_{m_{j}}\right)\left\|x_{m_{j}}-z_{0}\right\|^{2}+M_{1}\left(k_{m_{j}}-1\right)+M_{2} v_{m_{j}}+2 \alpha_{m_{j}}\left\langle x_{0}-z_{0}, x_{m_{j}+1}-z_{0}\right\rangle \\
\leqslant & \left(1-\alpha_{m_{j}}\right)\left\|x_{m_{j}}-z_{0}\right\|^{2}+M_{1}\left(k_{m_{j}}-1\right)+M_{2} v_{m_{j}}+2 \alpha_{m_{j}}\left\langle x_{0}-z_{0}, x_{m_{j}}-z_{0}\right\rangle \\
& +2 \alpha_{m_{j}}\left\|x_{0}-z_{0}\right\|\left\|x_{m_{j}+1}-x_{m_{j}}\right\| .
\end{aligned}
$$

It follows that

$$
\left\|x_{m_{j}}-z_{0}\right\|^{2} \leqslant \frac{1}{\alpha_{m_{j}}}\left[M_{1}\left(k_{m_{j}}-1\right)+M_{2} v_{m_{j}}\right]+2\left\langle x_{0}-z_{0}, x_{m_{j}}-z_{0}\right\rangle+2\left\|x_{0}-z_{0}\right\|\left\|x_{m_{j}+1}-x_{m_{j}}\right\| .
$$

In view of (3.29), (3.31), (3.34), and (iv), we have

$$
\lim _{j \rightarrow \infty}\left\|x_{m_{j}}-z_{0}\right\|=0 .
$$

Again apply (3.33) to obtain

$$
\begin{aligned}
\left\|x_{m_{j}+1}-z_{0}\right\|^{2} \leqslant & \left(1-\alpha_{m_{j}}\right)\left\|x_{m_{j}}-z_{0}\right\|^{2}+M_{1}\left(k_{m_{j}}-1\right)+M_{2} v_{m_{j}}+2\left\langle x_{0}-z_{0}, x_{m_{j}}-z_{0}\right\rangle \\
& +2\left\|x_{0}-u\right\|\left\|x_{m_{j}+1}-x_{m_{j}}\right\| .
\end{aligned}
$$

It follows from (3.29), (3.31), (3.35), (3.36), (i), and (iv) that

$$
\lim _{j \rightarrow \infty}\left\|x_{m_{j}+1}-u\right\|=0 .
$$

Applying Lemma 2.3, we obtain

$$
0 \leqslant\left\|x_{j}-z_{0}\right\| \leqslant\left\|x_{m_{j}+1}-z_{0}\right\| .
$$

Consequently, we get $x_{n} \rightarrow z_{0}$ as $n \rightarrow \infty$. The proof is completed.

Remark 3.5. Theorem 3.4 extends, improves and develops Theorem 2.1 and Theorem 2.2 of Qin et al. [16], Theorem 2.4 of Ge [7], Theorem 2.1 of Zegeye et al. [26], Theorem 2.1 of Olaleru and Okeke [14], and Theorem 9 of Takahashi and Yao [22] in the following aspects. 
- Theorem 3.4 extends, improves and develops corresponding results in [7, 14, 16, 22, 26] from the problem for finding an element of $\operatorname{Fix}(\mathrm{T})$ or $A^{-1} 0 \cap S^{-1}\left(B^{-1} 0\right)$ to the more general and challenging problem for finding an element of $\operatorname{Fix}(T) \cap A^{-1} 0 \cap S^{-1}\left(B^{-1} 0\right)$.

- We establish strong convergence results concerning asymptotically pseudo-contractive mappings in the intermediate sense by using the Ishikawa-like algorithm but not the hybrid algorithm. Therefore the computation of $C_{n} \bigcap Q_{n}$ is not required for each $n \geqslant 1$.

- The assumption that the interior of $\operatorname{Fix}(T)$ is empty is not required in our Theorem 3.4. However, it is very necessary in Theorem 2.1 of Zegeye et al. [26] and Theorem 2.1 of Olaleru and Okeke [14].

- The subset $\mathrm{C}$ of real Hilbert space $\mathrm{H}$ does not have to be bounded in our Theorem 3.4 which is indispensable in Theorem 2.1 and Theorem 2.2 of Qin et al. [16].

- The algorithm (3.3) is more advantageous and more flexible than the ones given in [16, 26]. In particular, whenever $E=H$, a Hilbert space, $A=B=0$ and $\alpha_{n} \equiv 0$, our scheme (3.3) reduces to (*) in [16] and (2.1) in [26]. Hence the new algorithm is expected to be widely applicable.

\section{An extension of our main results}

By using Theorem 3.4, we have the following strong convergence results for computing the fixed point problem of asymptotically pseudo-contractive mappings.

Conclusion 4.1. Let $E$ be a uniformly convex and smooth Banach space and let $J$ be the duality mapping on $\mathrm{E}$. Let $\mathrm{H}$ be a Hilbert space and let $\mathrm{C}$ be a nonempty closed convex subset of $\mathrm{H}$. Let $A$ and $\mathrm{B}$ be maximal monotone operators of $\mathrm{H}$ into $2^{\mathrm{H}}$ such that $\operatorname{dom}(A) \subset \mathrm{C}$ and $\mathrm{E}$ into $2^{\mathrm{E} *}$, respectively. Let $\mathrm{J}_{\lambda}$ be the resolvent of $A$ for $\lambda>0$ and let $Q_{\mu}$ be the metric resolvent of $B$ for $\mu>0$. Let $S: H \rightarrow E$ be a bounded linear operator such that $S \neq 0$ and let $S^{*}$ be the adjoint operator of $S$. Let $T: C \rightarrow C$ be a uniformly L-Lipschitz, uniformly asymptotically regular and asymptotically strict pseudo-contractive mapping in the intermediate sense with sequences $\left\{k_{n}\right\} \subset[1, \infty)$ and $\left\{v_{n}\right\} \subset[0, \infty)$ as defined in (1.2). Assume $\Gamma:=\operatorname{Fix}(T) \cap A^{-1} 0 \cap S^{-1}\left(B^{-1} 0\right)$ is nonempty and the element $x_{0} \in C$ is fixed. Let $\left\{x_{n}\right\}$ be a sequence generated by

$$
\left\{\begin{array}{l}
x_{1}, \text { chosen arbitrarily, } \\
z_{n}=J_{\lambda_{n}}\left(x_{n}-\delta_{n} S^{*} J\left(S x_{n}-Q_{\mu_{n}} S x_{n}\right)\right) \\
y_{n}=\left(1-\gamma_{n}\right) z_{n}+\gamma_{n} T^{n} z_{n}, \\
x_{n+1}=\alpha_{n} x_{0}+\left(1-\alpha_{n}\right)\left[\left(1-\beta_{n}\right) z_{n}+\beta_{n} T^{n} y_{n}\right]
\end{array}\right.
$$

where $\left\{\alpha_{n}\right\},\left\{\beta_{n}\right\},\left\{\gamma_{n}\right\} \subset[0,1]$ and $\left\{\lambda_{n}\right\},\left\{\mu_{n}\right\},\left\{\delta_{n}\right\} \subset(0,+\infty)$ satisfy the following conditions:

(i) $\lim _{n \rightarrow \infty} \alpha_{n}=0$ and $\sum_{n=1}^{\infty} \alpha_{n}=\infty$;

(ii) $0<\liminf _{n \rightarrow \infty} \beta_{n}$ and $\beta_{n} \leqslant \gamma_{n}$;

(iii) $a \leqslant \gamma_{n} \leqslant b$ for some $a>0$ and $b \in\left(0, \frac{1}{\sqrt{k_{n}^{2}+\mathrm{L}^{2}}+k_{n}}\right)$;

(iv) $\sum_{n=1}^{\infty} v_{n}<\infty, v_{n}=o\left(\alpha_{n}\right), k_{n}-1=o\left(\alpha_{n}\right)$ and $\sum_{n=1}^{\infty}\left(k_{n}-1\right)<\infty$;

(v) $0<\liminf _{n \rightarrow \infty} \delta_{n} \leqslant \limsup _{n \rightarrow \infty} \delta_{n}<\frac{2}{\|S\|^{2}}, 0<\liminf _{n \rightarrow \infty} \lambda_{n} \leqslant \limsup _{n \rightarrow \infty} \lambda_{n}<\infty$ and $0<\liminf _{n \rightarrow \infty} \mu_{n} \leqslant \limsup _{n \rightarrow \infty} \mu_{n}<\infty$.

Then the sequence $\left\{x_{n}\right\}$ generated by (4.1) converges strongly to a point $z_{0} \in \Gamma$, where $z_{0}=P_{\Gamma} x_{0}$.

Proof. Note that, any uniformly L-Lipschitzian and asymptotically k-strict pseudocontractive mapping $T$ in the intermediate sense is uniformly L-Lipschitzian and asymptotically pseudocontractive mapping in the intermediate sense and hence the conclusion follows from Theorem 3.4.

Conclusion 4.2. Let $\mathrm{H}$ be a Hilbert space and let $\mathrm{C}$ be a nonempty closed convex subset of $\mathrm{H}$. Let $A$ and $B$ be two maximal monotone operators of $H$ into $2^{\mathrm{H}}$ such that $\operatorname{dom}(A) \subset C$. Let $J_{\lambda}$ be the resolvent of $A$ for $\lambda>0$ and let $Q_{\mu}$ be the resolvent of $B$ for $\mu>0$. Let $S: H \rightarrow H$ be a bounded linear operator such 
that $S \neq 0$ and let $S^{*}$ be the adjoint operator of $S$. Let $T: C \rightarrow C$ be a uniformly L-Lipschitz, uniformly asymptotically regular and asymptotically pseudo-contractive mapping in the intermediate sense with sequences $\left\{k_{n}\right\} \subset[1, \infty)$ and $\left\{v_{n}\right\} \subset[0, \infty)$ as defined in (1.4). Assume $\Gamma:=\operatorname{Fix}(T) \cap A^{-1} 0 \bigcap S^{-1}\left(B^{-1} 0\right)$ is nonempty and the element $x_{0} \in C$ is fixed. Let $\left\{x_{n}\right\}$ be a sequence generated by

$$
\left\{\begin{array}{l}
x_{1}, \text { chosen arbitrarily, } \\
z_{n}=J_{\lambda_{n}}\left(x_{n}-\delta_{n} S^{*}\left(S x_{n}-Q_{\mu_{n}} S x_{n}\right)\right) \\
y_{n}=\left(1-\gamma_{n}\right) z_{n}+\gamma_{n} T^{n} z_{n}, \\
x_{n+1}=\alpha_{n} x_{0}+\left(1-\alpha_{n}\right)\left[\left(1-\beta_{n}\right) z_{n}+\beta_{n} T^{n} y_{n}\right]
\end{array}\right.
$$

where $\left\{\alpha_{n}\right\},\left\{\beta_{n}\right\},\left\{\gamma_{n}\right\} \subset[0,1]$ and $\left\{\lambda_{n}\right\},\left\{\mu_{n}\right\},\left\{\delta_{n}\right\} \subset(0,+\infty)$ satisfy the following conditions:

(i) $\lim _{n \rightarrow \infty} \alpha_{n}=0$ and $\sum_{n=1}^{\infty} \alpha_{n}=\infty$;

(ii) $0<\liminf _{n \rightarrow \infty} \beta_{n}$ and $\beta_{n} \leqslant \gamma_{n}$;

(iii) $a \leqslant \gamma_{n} \leqslant b$ for some $a>0$ and $b \in\left(0, \frac{1}{\sqrt{k_{n}^{2}+\mathrm{L}^{2}}+k_{n}}\right)$;

(iv) $\sum_{n=1}^{\infty} v_{n}<\infty, v_{n}=o\left(\alpha_{n}\right), k_{n}-1=o\left(\alpha_{n}\right)$ and $\sum_{n=1}^{\infty}\left(k_{n}-1\right)<\infty$;

(v) $0<\liminf _{n \rightarrow \infty} \delta_{n} \leqslant \limsup _{n \rightarrow \infty} \delta_{n}<\frac{2}{\|S\|^{2}}, 0<\liminf _{n \rightarrow \infty} \lambda_{n} \leqslant \limsup _{n \rightarrow \infty} \lambda_{n}<\infty$ and $0<\liminf _{n \rightarrow \infty} \mu_{n} \leqslant \limsup _{n \rightarrow \infty} \mu_{n}<\infty$.

Then the sequence $\left\{x_{n}\right\}$ generated by (4.2) converges strongly to a point $z_{0} \in \Gamma$, where $z_{0}=P_{\Gamma} x_{0}$.

\section{Acknowledgment}

This research was partially supported by the Innovation Program of Shanghai Municipal Education Commission (15ZZ068) and Ph.D. Research Foundation of Zhongyuan University of Technology (2200/34110323).

\section{References}

[1] Y. I. Alber, Metric and generalized projections operators in Banach spaces: properties and applications, Dekker, New York, (1996). 2

[2] K. Aoyama, F. Kohsaka, W. Takahashi, Three generalizations of firmly nonexpansive mappings: their relations and continuous properties, J. Nonlinear Convex Anal., 10 (2009), 131-147. 2, 3.1

[3] V. Barbu, Nonlinear Semigroups and Differential Equations in Banach spaces, Noordhoff International Publishing, Leiden, (1976). 3.3

[4] F. E. Browder, Nonlinear maximal monotone operators in Banach spaces, Math. Ann., 175 (1968), 89-113. 2

[5] S. Y. Cho, Strong convergence analysis of a hybrid algorithm for nonlinear operators in a Banach space, J. Appl. Anal. Comput., 8 (2018), 19-31. 1

[6] J. Deepho, P. Thounthong, P. Kumam, S. Phiangsungnoen, A new general iterative scheme for split variational inclusion and fixed point problems of $k$-strict pseudo-contraction mappings with convergence analysis, J. Comput. Appl. Math., 318 (2017), 293-306. 1

[7] C.-S Ge, A hybrid algorithm with variable coefficients for asymptotically pseudocontractive mappings in the intermediate sense on unbounded domains, Nonlinear Anal., 75 (2012), 2859-2866. 1.2, 1, 1.3, 1, 3.5

[8] K. Goebel, W. A. Kirk, A fixed point theorem for asymptotically nonexpansive mappings, Proc. Amer. Math. Soc., 35 (1972), 171-174. 1

[9] T.-H. Kim, H.-K. Xu, Convergence of the modified Mann's iteration method for asymptotically strict pseudo-contractions, Nonlinear Anal., 68 (2008), 2828-2836. 1.1

[10] Q. H. Liu, Convergence theorems of the sequence of iterates for asymptotically demicontractive and hemicontractive mappings, Nonlinear Anal., 26 (1996), 1835-1842. 1

[11] L. S. Liu, Ishikawa and Mann iterative process with errors for nonlinear strongly accretive mappings in Banach spaces, J. Math. Anal. Appl., 194 (1995), 114-125. 2.7

[12] P.-E. Mainge, Approximation methods for common fixed points of nonexpansive mappings in Hilbert spaces, J. Math. Anal. Appl., 325 (2007), 469-479. 2.3

[13] A. Moudafi, Krasnoselski-Mann iteration for hierarchical fixed-point problems, Inverse Problems, 23 (2007), $1635-1640$. 1 
[14] J. O. Olaleru, G. A. Okeke, Strong convergence theorems for asymptotically pseudocontractive mappings in the intermediate sense, British J. Math. Computer Sci., 2 (2012), 151-162. 1, 3.5

[15] X. Qin, S. Y. Cho, Convergence analysis of a monotone projection algorithm in reflexive Banach spaces, Acta Math. Sci. Ser. B (Engl. Ed.), 37 (2017), 488-502. 1

[16] X. Qin, S. Y. Cho, J. K. Kim, Convergence theorems on asymptotically pseudocontractive mappings in the intermediate sense, Fixed Point Theory Appl., 2010 (2010), 14 pages. 1, 1, 1, 2.5, 2.6, 3.5

[17] X. Qin, J.-C. Yao, Projection splitting algorithms for nonself operators, J. Nonlinear Convex Anal., 18 (2017), $925-935$. 1

[18] D. R. Sahu, H.-K. Xu, J.-C. Yao, Asymptotically strict pseudocontractive mappings in the intermediate sense, Nonlinear Anal., 70 (2009), 3502-3511. 1

[19] J. Schu, Iterative construction of fixed points of asymptotically nonexpansive mapping, J. Math. Anal. Appl., 158 (1991), 407-413. 1, 1

[20] W. Takahashi, Convex Analysis and Approximation of Fixed Points, Yokohama Publ., Yokohama, (2000). 2

[21] W. Takahashi, Nonlinear Functional Analysis, Yokohama Publ., Yokohama, (2000). 2

[22] W. Takahashi, J.-C. Yao, Strong convergence theorems by hybrid methods for the split common null point problem in Banach spaces, Fixed Point Theory Appl., 2015 (2015), 13 pages. 1, 1, 3.1, 3.5

[23] K.-K. Tan, H.-K. Xu, Approximating fixed points of nonexpansive mappings by the Ishikawa iteration process, J. Math. Anal. Appl., 178 (1993), 301-308. 2.4

[24] P. Tianchai, An approximate solution to the fixed point problems for an infinite family of asymptotically strictly pseudocontractive mappings in the intermediate sense, cocoercive quasivariational inclusions problems and mixed equilibrium problems in Hilbert spaces, Fixed Point Theory Appl., 2012 (2012), 26 pages. 1

[25] I. Yamada, N. Ogura, Hybrid steepest descent method for the variational inequality problem over the fixed point set of certain quasi-nonexpansive mappings, Numer. Funct. Anal. Optim., 25 (2004), 619-655. 1

[26] H. Zegeye, M. Robdera, B. Choudhary, Convergence theorems for asymptotically pseudocontractive mappings in the intermediate sense, Comput. Math. Appl., 62 (2011), 326-332. 1, 1, 3.5

[27] H. Zhou, Demiclosedness principle with applications for asymptotically pseudo-contraction in Hilbert spaces, Nonlinear Anal., 70 (2009), 3140-3145. 1, 1 